\title{
Large-scale identification of differentially expressed genes during pupa development reveals solute carrier gene is essential for pupal pigmentation in Chilo suppressalis
}

\author{
Yang Sun $^{\mathrm{a}, 1}$, Shuijin Huang ${ }^{\mathrm{b}, 1}$, Shuping Wang ${ }^{\mathrm{c}}$, Dianhao Guo ${ }^{\mathrm{a}}$, Chang Ge ${ }^{\mathrm{a}}$, Huamei Xiao ${ }^{\mathrm{a}}$, Wencai Jie ${ }^{\mathrm{a}}$, \\ Qiupu Yang ${ }^{\mathrm{a}}$, Xiaolu Teng ${ }^{\mathrm{a}}$, Fei $\mathrm{Li}^{\mathrm{a}, \mathrm{d}, *}$ \\ a Department of Entomology, Nanjing Agricultural University, Nanjing 210095, China \\ b Institute of Plant Protection, Jiangxi Academy of Agricultural Science, Nanchang 330200, China \\ ${ }^{\mathrm{c}}$ Technical Centre for Animal Plant and Food Inspection and Quarantine, Shanghai Entry-exit Inspection and Quarantine Bureau, Shanghai 200135, China \\ ${ }^{\mathrm{d}}$ Ministry of Agriculture Key Lab of Agricultural Entomology, Institute of Insect Sciences, Zhejiang University, 866 Yuhangtang Road, Hangzhou 310058, China
}

\section{A R T I C L E I N F O}

Article history:

Received 6 August 2016

Received in revised form 22 November 2016

Accepted 27 December 2016

Available online 29 December 2016

\section{Keywords:}

Chilo suppressalis

Pupa development

Microarray

Gene expression profile

RNA interference

Solute carrier genes

\begin{abstract}
A B S T R A C T
Insects undergo metamorphosis, involving an abrupt change in body structure through cell growth and differentiation. Rice stem stripped borer (SSB), Chilo suppressalis, is one of the most destructive rice pests. However, little is known about the regulation mechanism of metamorphosis development in this notorious insect pest. Here, we studied the expression of 22,197 SSB genes at seven time points during pupa development with a customized microarray, identifying 622 differentially expressed genes (DEG) during pupa development. Gene ontology (GO) analysis of these DEGs indicated that the genes related to substance metabolism were highly expressed in the early pupa, which participate in the physiological processes of larval tissue disintegration at these stages. In comparison, highly expressed genes in the late pupal stages were mainly associated with substance biosynthesis, consistent with adult organ formation at these stages. There were 27 solute carrier $(S L C)$ genes that were highly expressed during pupa development. We knocked down SLC22A3 at the prepupal stage, demonstrating that silencing SLC22A3 induced a deficiency in pupa stiffness and pigmentation. The RNAi-treated individuals had white and soft pupa, suggesting that this gene has an essential role in pupal development.
\end{abstract}

(c) 2016 Elsevier Ltd. All rights reserved.

\section{Introduction}

Insect development is characterized by metamorphosis that involves abrupt changes in body structures. It has been reported that the balance between ecdysteroid and juvenile hormone $(\mathrm{JH})$ is crucial in regulating metamorphosis (Cho et al., 2014; Jindra et al., 2013; Yamanaka et al., 2013). The orchestrated actions of ecdysteroid and $\mathrm{JH}$, and their downstream physiological responses regulate development during metamorphosis (Riddiford, 2012). In the process of transforming from larvae to adult, two developmental programs are activated by ecdysteroid in pupa, the destruction of larval tissues by programmed cell death and the formation of adult tissues by cell differentiation (Yin and Thummel, 2005).

\footnotetext{
* Corresponding author at: Department of Entomology, Nanjing Agricultural University, Nanjing 210095, China.

E-mail address: lifei18@zju.edu.cn (F. Li).

1 The first two authors contribute equally and should be treated as joint first authors.
}

The regulation mechanism of pupa development has been well studied in Drosophila melanogaster, Bombyx mori, Tribolium castaneum, etc (Jindra et al., 2013; Riddiford, 2007, 2008, 2012; Truman and Riddiford, 2007). As a model organism, D. melanogaster serves as an important system to identify genes involved in metamorphosis development (Jones et al., 2010). JH has a key role in the prepupal development of $D$. melanogaster (Riddiford et al., 2010). Many genes such as protein tyrosine phosphatase PTP52F was confirmed to be indispensable for larva-pupa transition in Drosophila (Santhanam et al., 2013). The silkworm B. mori is another model insect which has been well studied to uncover the mechanism of metamorphosis. As well known, JH also has a key role in larval-pupal transformation of the cecropia silkworm (Riddiford, 1972). Fifty-seven genes were specifically expressed in the intersegmental muscle of larvae whereas another 54 genes were in the pupa of $B$. mori. Twelve proteins were also found to be significantly upregulated during pupation in $B$. mori (Zhang et al., 2007a). A cDNA microarray analysis indicated that the expression of Bombyx genes were clustered into 12 patterns from 
the first instar larvae to pupa (Zhang et al., 2007a,b). Homeodomain proteins participate in regulation of embryonic development. A study indicated that they also regulate the larval-to-pupal transition by interacting with another homeodomain transcription factor, BmAbd-A (Deng et al., 2012).

Recently, RNA sequencing (RNA-Seq) has been used to screen genes associated with developments in several non-model insects. In $T$. castaneum, a large-scale and unbiased iBeetle screen identified many novel genes participating in oogenesis, embryogenesis and metamorphosis (Schmitt-Engel et al., 2015), which is a good supplement to Drosophila study. Transcriptome analysis of wing disc from 6-day-old fifth instar larvae (L5D6), prepupae (PP) and pupae (P0) identified thousands of genes invovled in larva-to-pupa metamorphosis development (Ou et al., 2014). A transcriptome-wide analysis of gene expression of five cycle stages of bumblebee Bombus terrestris showed that genes involved in amino acid storage, metabolism, immunity and olfaction were differentially expressed (Colgan et al., 2011). Developmental transcriptome analysis of the small white butterfly Pieris rapae yielded 849 genes that involved in seven canonical development signaling pathways (Qi et al., 2016). Similar strategy has also been applied to study Athetis lepigone, an important insect pest of maize (Li et al., 2013), and the synanthropic fly Chrysomya megacephala (Wang et al., 2015), finding hundreds of or thousands of differentially expressed genes invovled in larvae or pupa development.

Rice striped stem borer (SSB), Chilo suppressalis Walker, is one of the most notorious and destructive rice pests. It is widely distributed from Asia to the Pacific, causing huge rice yield losses (He et al., 2013). Here, we used a customized microarray to study the gene expressions at seven developmental time-points during pupal development of SSB, identifying 622 differentially expressed genes. SLC22A3 was selected for further study and it was shown to have an essential role in pupal pigmentation in SSB.

\section{Materials and methods}

\subsection{Insect rearing and specimen preparation}

Insects were collected from a rice field in Cangnan County in Zhejiang Province, China. The insects were kept on rice seedlings at $28 \pm 1{ }^{\circ} \mathrm{C}$ under a 16 -h photoperiod and $>80 \%$ relative humidity. For microarray analysis, insects at seven developmental timepoints during pupal development were selected, including aging larval (aL), prepupal (pP), early pupal (eP), compound eye formation (cE), pretarsal formation (pT), pupal elongation (Pe), and adult (Ad) stages (Fig. 1). aL is late larvae which stop eating and begin to find dry place for pupation. $\mathrm{pP}$ is the insects at wandering stage. $\mathrm{eP}$ is early pupae within 10 min and the colour of the pupae is still white. $\mathrm{cE}$ is the pupa whose compound eye became brown under the dissecting microscope. pT is the pupa when pretarsus can be observed under the dissecting microscope. Pe is the pupa just before molting, when the pupae become golden and much longer. Ad is the adult molting within $8 \mathrm{~h}$. Four individuals (two males and two females) were used for each time-point as one biological replicate. There is a "Y" shaped feature at the abdomen end of female pupae but not male. By this feature, we can identify females and males. Insects were frozen in liquid nitrogen and stored at $-70{ }^{\circ} \mathrm{C}$ before use. All of the experiments were repeated in triplicate.

\subsection{Microarray probes design}

We developed a transcriptome of SSB (SRA050703) (Yin et al., 2014), producing 22,197 unigenes by using Trinity with the default parameters (Grabherr et al., 2011). The contigs were annotated by using BLASTX against the GenBank non-redundant (nr) database with an E-value cutoff set to $10^{-5}$. We designed a customized standard $12 \times 135 \mathrm{~K}$ microarray with a NimbleGen hybridisation system (Roche, Swiss) to study the expression of 22,197 SSB unigenes. Six different probes that ranged from 45- to 60-mers were designed based on the sequences of unigenes.

\subsection{Microarray experiment}

The microarray experiment was carried out following the procedure described previously (Guo et al., 2005) with a little modification. All experiments were carried out in CapitalBio Co. Ltd (Beijing, China).

\subsection{1. $C R N A$ preparation}

Total RNA was extracted using Trizol reagent and purified using NucleoSpin ${ }^{\circledR}$ RNA clean-up (Macherey-Nagel, Germany). Eberwine's linear RNA amplification method and subsequent enzymatic reaction was used for cDNA synthesis and labelled with a fluorescent dye (Cy3-dCTP). The CapitalBio cRNA Amplification and Labelling Kit (CapitalBio, China) were used for cRNA preparation.

\subsection{2. cDNA synthesis and labelling}

Briefly, $2 \mu \mathrm{g}$ amplified RNA was mixed with $4 \mu \mathrm{g}$ random nanomer, denatured at $65^{\circ} \mathrm{C}$ for $5 \mathrm{~min}$, and cooled on ice. Then, $5 \mu \mathrm{L}$ of $4 \times$ first-strand buffer, $2 \mu \mathrm{L}$ of $0.1 \mathrm{M}$ DTT, and $1.5 \mu \mathrm{L}$ CbcScript II reverse transcriptase were added. The mixture was incubated at $25^{\circ} \mathrm{C}$ for $10 \mathrm{~min}$, then at $37{ }^{\circ} \mathrm{C}$ for $90 \mathrm{~min}$. The cDNA products were purified using a PCR NucleoSpin Extract II Kit (Macherey-Nagel) and vacuum evaporated to $14 \mu \mathrm{L}$. The cDNA was mixed with $4 \mu \mathrm{g}$ random nanomer, heated to $95^{\circ} \mathrm{C}$ for $3 \mathrm{~min}$, and snap cooled on ice for $5 \mathrm{~min}$. Then, $5 \mu \mathrm{L}$ Klenow buffer, dNTP, and Cy3-dCTP (GE Healthcare, United Kingdom) were added to final concentrations of $240 \mu \mathrm{M}$ dATP, $240 \mu \mathrm{M}$ dGTP, $240 \mu \mathrm{M}$ dTTP, $120 \mu \mathrm{M}$ dCTP, and $40 \mu \mathrm{M}$ Cy-dCTP. Next, $1.2 \mu \mathrm{L}$ Klenow enzyme was added, and the reaction was performed at $37^{\circ} \mathrm{C}$ for $90 \mathrm{~min}$. Labelled cDNA was purified with a PCR NucleoSpin Extract II Kit (Macherey-Nagel) and re-suspended in elution buffer.

\subsubsection{Hybridisation, washing, and scanning}

The experiments were carried out according to the NimbleGen Expression user's guide and performed at CapitalBio Corporation (Beijing, China). Labelled samples were dried and re-dissolved in the hybridisation solution. Hybridization was performed at $42^{\circ} \mathrm{C}$ for $14 \mathrm{~h}$ with NimbleGen Hybridization System, Array were washed in wash buffer I and II and III supplied by NimbleGen and dried in NimbleGen Microarray Dryer. After drying, the arrays were scanned using an MS200 scanner (NimbleGen) with $2 \mu \mathrm{m}$ resolution, and NimbleScan software (NimbleGen) was used to extract the fluorescent intensity raw data from the scanned images (see manufacturer's user guide).

\subsection{Data analysis}

The expression data of the probes were normalized using quantile normalisation (Bolstad et al., 2003) and expression data of the genes were generated using the Robust Multichip Average (RMA) algorithm with default parameters (Irizarry et al., 2003a,b). To identify differentially expressed genes (DEGs), Significant Analysis of Microarray (SAM) software (version 3.02) (Tusher et al., 2001) was used with a threshold of false discovery rate of $<5 \%$. The cutoff was set as q-value (\%) $=0$ and fold change $\geqslant 2$. Hierarchical clustering with the average linkage method was performed with Cluster 3.0 software (Eisen et al., 1998) and cluster results were visualised with the TreeView program (Eisen et al., 1998). Insect 


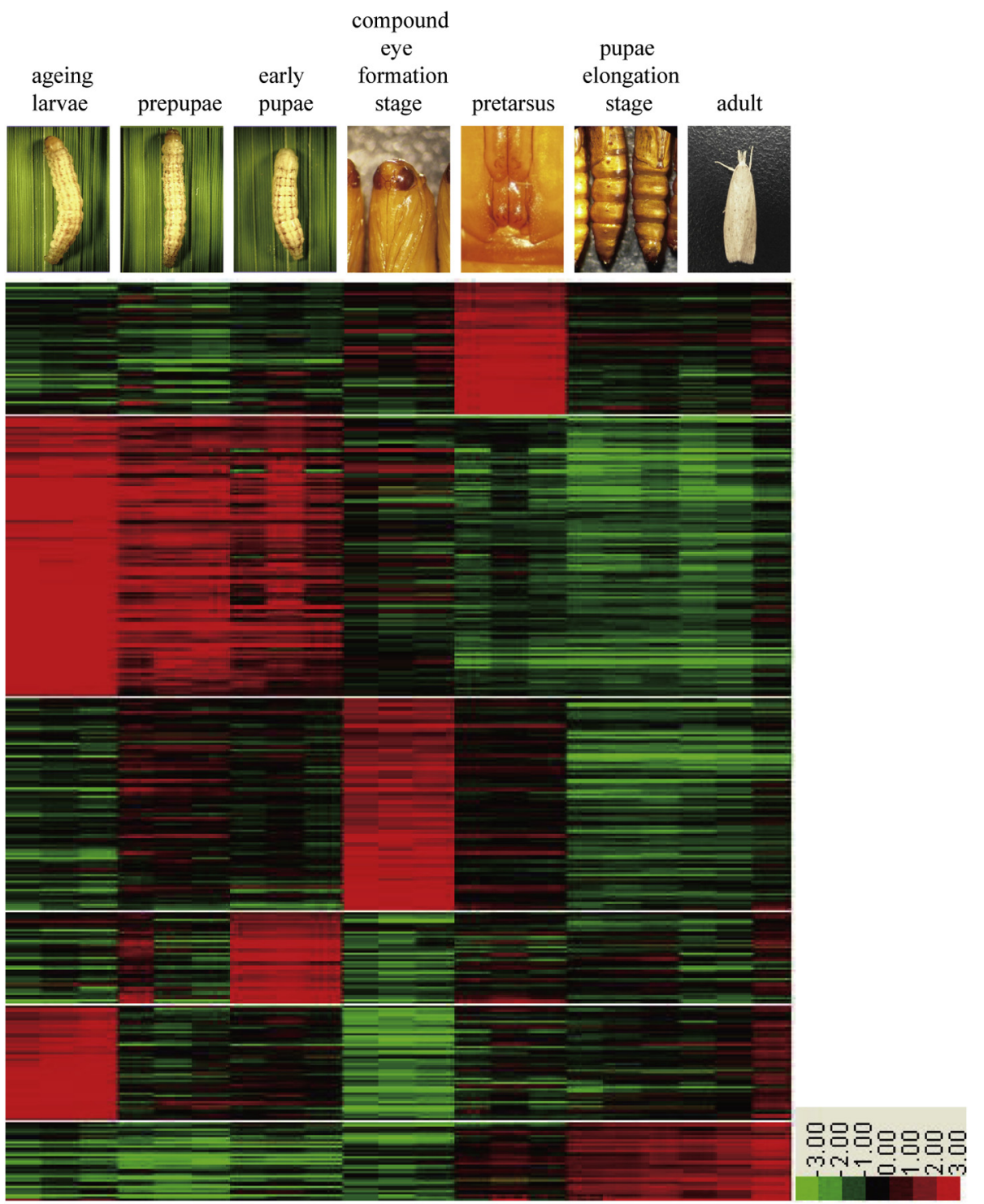

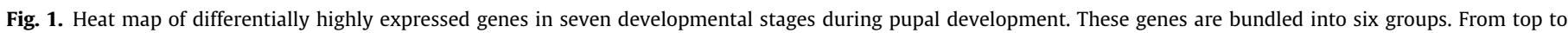

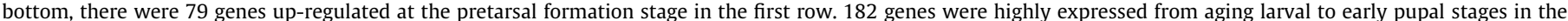

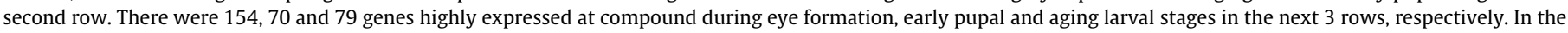
last row, 58 genes were highly expressed in the pupal elongation and adult stages.

pathway analysis was conducted using iPathCons in InsectBase (Yin et al., 2016; Zhang et al., 2014).

\subsection{RT-PCR and $q P C R$}

Total RNA was isolated using Trizol reagent followed by RNase free DNase I treatment (Roche Diagnostics, Mannheim, Germany). The cDNA was synthesised using PrimeScript ${ }^{\circledR}$ RT Master Mix kit (Takara, Japan) with oligo (dT) and 500 ng total RNA. The primers for reverse-transcription PCR (RT-PCR) were designed using Primer Premier 5.0 and are found in Table S1. Premix Taq ${ }^{\circledR}$ Version 2.0 kit (Takara) was used for the PCR reactions. The primers for quantitative real time PCR (qPCR) were designed with an online tool (http://www.idtdna.com/Scitools/) and synthesised in GenScript (Nanjing, China). The primer sequences are found in Table S1. The qPCR reactions were performed using an ABI 7300 Real-Time PCR System (Applied Biosystems, Foster City, CA). A SYBR Premix Ex Taq kit (Takara) was used with a standard PCR protocol of denaturing at $95^{\circ} \mathrm{C}$ for $30 \mathrm{~s}$, followed by 40 cycles of $95^{\circ} \mathrm{C}$ for $5 \mathrm{~s}$ and $60{ }^{\circ} \mathrm{C}$ for $31 \mathrm{~s}$. Melting curves were determined by heating the sam- ple to $95{ }^{\circ} \mathrm{C}$ for $15 \mathrm{~s}$, followed by $60{ }^{\circ} \mathrm{C}$ for $15 \mathrm{~s}$, and again heating to $95^{\circ} \mathrm{C}$ for $15 \mathrm{~s}$. The actin gene was used as the internal control. Data were analyzed using the $2^{-\Delta \Delta \mathrm{Ct}}$ method (Pfaffl, 2001).

\subsection{RNA interference}

The siRNAs used in the RNAi experiments were chemically synthesised by GenePharma Co., Ltd. (Shanghai, China). The siRNA sequences are found in Table S1. The double-stranded siRNAs were purified by high-performance liquid chromatography, and dissolved in diethylpyrocarbonate-treated water (Milli-Q-grade) to a final concentration of $4 \mathrm{mg}$ siRNA/mL $\mathrm{H}_{2} \mathrm{O}$. Then $1 \mu \mathrm{L}(4 \mu \mathrm{g})$ of siRNA was injected into the larvae or pupa using a microneedle. The needles were pulled from glass capillaries $(1.0 \mathrm{~mm}$ outer diameter and $0.50 \mathrm{~mm}$ inner diameter) using a micropipette puller (Model P-87, Sutter Instruments Co., Novato, CA). To avoid leakage of siRNAs, needles were held still at the injection point for $30 \mathrm{~s}$. The shuffled siRNAs were used as the negative control. Thirty insects were used for each treatment and all of the experiments were per- 
formed in triplicate. A $t$-test was used for statistical analysis and the significance level was set to a p-value of $<0.05$.

\section{Results}

\subsection{Gene expression patterns during pupal development}

A customized NimbleGen microarray was used to examine the expressions of all 22,197 SSB transcripts at seven developmental time-points during pupal development (Fig. 1). All seven stages were analyzed with three biological repeats. An unsupervised clustering showed biological repeats of different stages had high reproducibility (Fig. S1). The results indicated that gene expression in pupal development could be classified into two subgroups. One is an early pupal subgroup, consisting of aging larval, prepupal, early pupal, and compound eye formation stages. These four stages shared similar gene expression patterns. The other is a late pupal subgroup, including pretarsal formation, pupal elongation and adult stages. The gene expressions of these three stages were clustered together. This result is consistent with the present understanding of pupal development. The larvae tissues are disintegrated during early pupal stages whereas adult organs form in the late pupal stages.

\subsection{Differentially expressed genes in pupal development}

To identify DEGs in pupal development, the ratio of the highest signal value (rank1) to the second highest (rank2) was calculated for each gene. When the cutoff was set with a q-value $=0$ and a ratio $>2$, there were 622 DEGs at different developmental timepoints. Of them, $79,70,154$, and 79 genes were highly expressed at aging larval, early pupal, compound eye formation, and pretarsal formation stages, respectively (Figs. 1 \& 2). There were 182 genes co-expressed highly at aging larval, prepupal, and early pupal stages, while 58 genes were co-expressed highly at the pupal elongation and adult stages (Fig. 2). The differentially expressed genes at each developmental stage are listed in Supplemental file 1.

Among these genes, chitinases and cuticle proteins genes were upregulated at aging larval and early pupal stages. Hormone receptor 4 (HR4), a central regulator of genetic responses to ecdysteroid at the onset of metamorphosis (King-Jones et al., 2005), was highly expressed at the compound eye formation stage. The functions of BR-C, E74, FTZ-F1 and Kr-h1 have been well studied in the metamorphosis development (Kayukawa et al., 2014; Stilwell et al., 2003; Sultan et al., 2014). We examined the expression patterns of these genes from microarray data and found that they were in general consistent with previous reports, showing the microarray data was reliable for further analysis. Among which, $B R-C$ highly expressed from aging larvae and decreased rapidly after pupation, which is consistent with the expressional pattern in the red flour beetle and silkworm (Parthasarathy et al., 2008; Reza et al., 2004). E74 highly expressed around pupation. FTZ-F1 was expressed throughout the pupa, with especially high-expression the late pupal stage. The transcript of $K r$ - $h 1$ was abundant at the prepupal stage but was undetectable from early pupae to middle pupae. It gradually increased from late pupae to adult, and the transcript level was the highest at adult stage (Fig. S2).

Pathway analysis of DEGs was conducted using iPathCons (Zhang et al., 2014). In total, 83 pathways were identified, 22 of which could be classified into three categories of energy metabolism, sensory system and hormone-related pathways (Table S2). The highly expressed genes at the aging larval, prepupal and early pupal stages mainly involved protein digestion and absorption (ko04974), amino sugar and nucleotide sugar metabolism (ko00520), and carbohydrate digestion and absorption (ko04973). These pathways are closely associated with substance metabolism, which is consistent with the physiological process of tissue disintegration at these stages. In contrast, the highly expressed genes

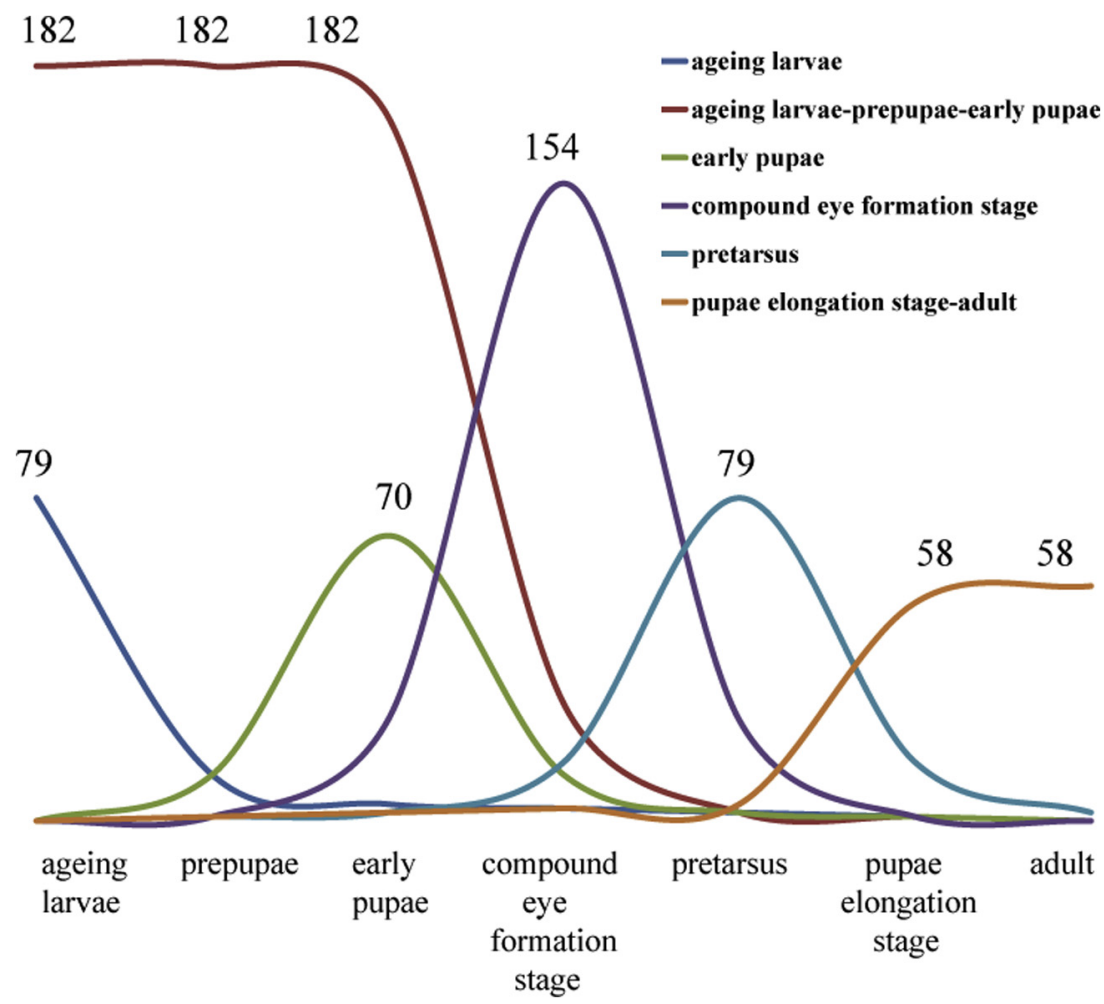

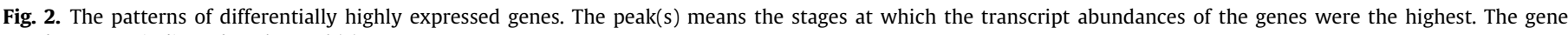
numbers were indicated at the peak(s). 
at the pupal elongation and adult stages were signal transduction pathways such as taste transduction (ko04742), phototransduction (ko04744), retinol metabolism (ko00830) and carotenoid biosyn-
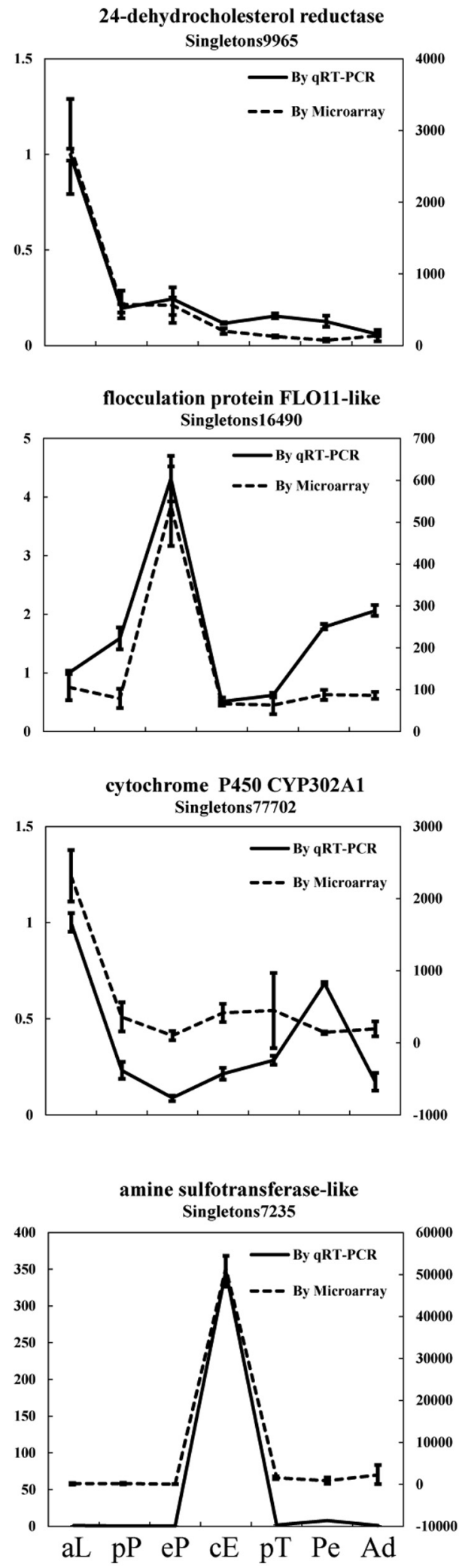

Development Stages thesis (ko00906). There were 9 DEGs in hormone-related pathways. We selected them for $\mathrm{qPCR}$ validation and the expression of eight genes were consistent with the microarray data (Fig. 3).
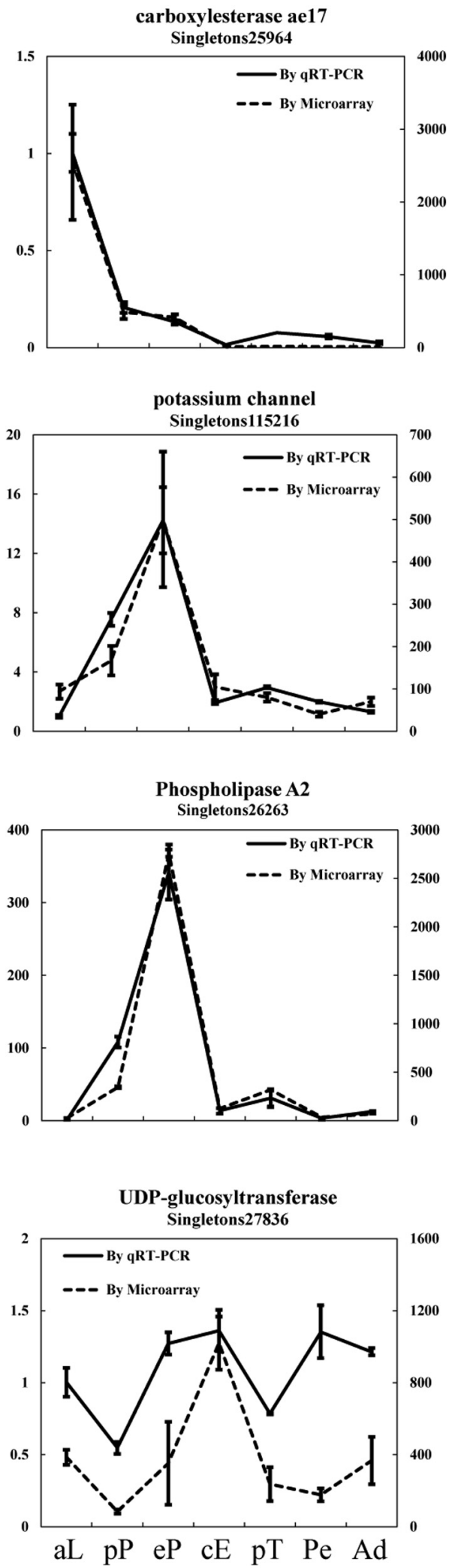

Development Stages

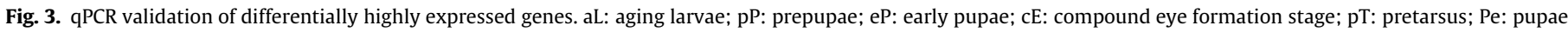
elongation stage; Ad: adult. Error bars indicate standard deviation (s. d.). 


\subsection{Knockdown of SLC22A3 led to white and soft pupa}

Among the list of DEGs, there were 27 solute carrier $(S L C)$ genes (Table 1). Among these SLC genes, SLC22A3 was the most highly expressed gene at the early pupal stage with a ratio of 39.62 . SLC22 is known to participate in the absorption and excretion of drugs, xenobiotic, and endogenous compounds in mammal intestines, liver, and/or kidney (Pelis and Wright, 2014). We chose CsuSLC22A3 to study its function in pupal development of SSB. Phylogenetic analysis of this gene with other SLCA subfamily genes indicated that CsuSLC22A3 shared high relatedness with the homo- logs in other lepidopteran insects (Fig. S3). Pfam domain analysis showed that CSUSLC22A3 had the conserved domains of SLC gene family such as major facilitator superfamily-1 (MSF_1) and Sugar (and other) transporter (Sugar_tr) (Fig. S4).

We used qPCR to examine the expression of CsuSLC22A3 during larval, pupa and adult development, suggesting that the highest expression appeared at the fifth day in the fifth instar larvae (Fig. 4). It was highly expressed at the early pupa stage, which was consistent with the microarray analysis (Fig. 5a). We successfully knocked down CSUSLC22A3 gene by injecting small interfering RNA (siRNA) at the prepupal stage. The transcript abundance of

Table 1

Differentially expressed solute carrier family genes in different stages.

\begin{tabular}{|c|c|c|c|}
\hline Development Stages & GeneID & Ratio & Homologous genes in blastx results \\
\hline \multirow[t]{4}{*}{ Aging larvae } & Singletons 104470 & 6.87 & Solute carrier family 22 member 2 (SLC22A2) \\
\hline & Singletons 24463 & 5.59 & Solute carrier family 22 member 15 (SLC22A35) \\
\hline & Singletons 28023 & 2.55 & Solute carrier family 2 , facilitated glucose transporter member 8 (SLC2A8) \\
\hline & Singletons9176 & 2.22 & Proton-coupled amino acid transporter 1 (SLC36A1) \\
\hline \multirow{4}{*}{$\begin{array}{l}\text { Aging larvae- } \\
\text { prepupae- } \\
\text { early pupae }\end{array}$} & Singletons21491 & 5.66 & Proton-coupled amino acid transporter 1 (SLC36A1) \\
\hline & Singletons 8035 & 3.03 & Organic cation transporter protein (Orct) \\
\hline & Singletons3013 & 2.50 & Solute carrier family 22 member 3 (SLC22A3) \\
\hline & Singletons 81470 & 2.42 & Organic cation transporter-like protein (Orct2) \\
\hline \multirow[t]{4}{*}{ Early pupae } & Singletons 102752 & 39.62 & Solute carrier family 22 member 3 (SLC22A3) \\
\hline & Singletons 23617 & 2.67 & Organic cation transporter protein (Orct) \\
\hline & Singletons 110766 & 2.60 & Sodium-independent sulfate anion transporter (SLC26A11) \\
\hline & Singletons 24544 & 2.11 & Solute carrier family 2 , facilitated glucose transporter member 8 (SLC2A8) \\
\hline Compound eye formation stage & Singletons8003 & 2.05 & Solute carrier family 46 member 3 (SLC46A3) \\
\hline \multirow[t]{7}{*}{ Pretarsus } & Singletons99734 & 7.64 & Sodium-independent sulfate anion transporter (SLC26A11) \\
\hline & Singletons 110102 & 6.65 & Sodium-independent sulfate anion transporter (SLC26A11) \\
\hline & Singletons114116 & 6.58 & Solute carrier family 2 , facilitated glucose transporter member 8 (SLC2A8) \\
\hline & Singletons114849 & 5.98 & Solute carrier family 2 , facilitated glucose transporter member 8 (SLC2A8) \\
\hline & Singletons113765 & 5.50 & Solute carrier family 22 member 11 (SLC22A31) \\
\hline & Singletons 10224 & 3.88 & Sodium-independent sulfate anion transporter (SLC26A11) \\
\hline & Singletons27909 & 2.47 & Long-chain fatty acid transport protein 4 (SLC27A4) \\
\hline \multirow[t]{7}{*}{ Pupae elongation stage-adult } & Singletons15995 & 7.83 & Solute carrier family 2 , facilitated glucose transporter member 2 (SLC2A2) \\
\hline & Singletons 24811 & 3.97 & Monocarboxylate transporter 6 (SLC16A5) \\
\hline & Singletons78036 & 2.95 & Zinc transporter 8 (SLC30A8) \\
\hline & Singletons 23250 & 2.90 & Monocarboxylate transporter 13 (SLC16A13) \\
\hline & Singletons 109185 & 2.77 & Solute carrier family 2 , facilitated glucose transporter member 8 (SLC2A8) \\
\hline & Singletons 26902 & 2.65 & Organic cation transporter protein $(\mathrm{Orct})$ \\
\hline & Singletons115293 & 2.49 & Monocarboxylate transporter 9 (SLC16A9) \\
\hline
\end{tabular}

\section{Expression Profile of SLC22A3}

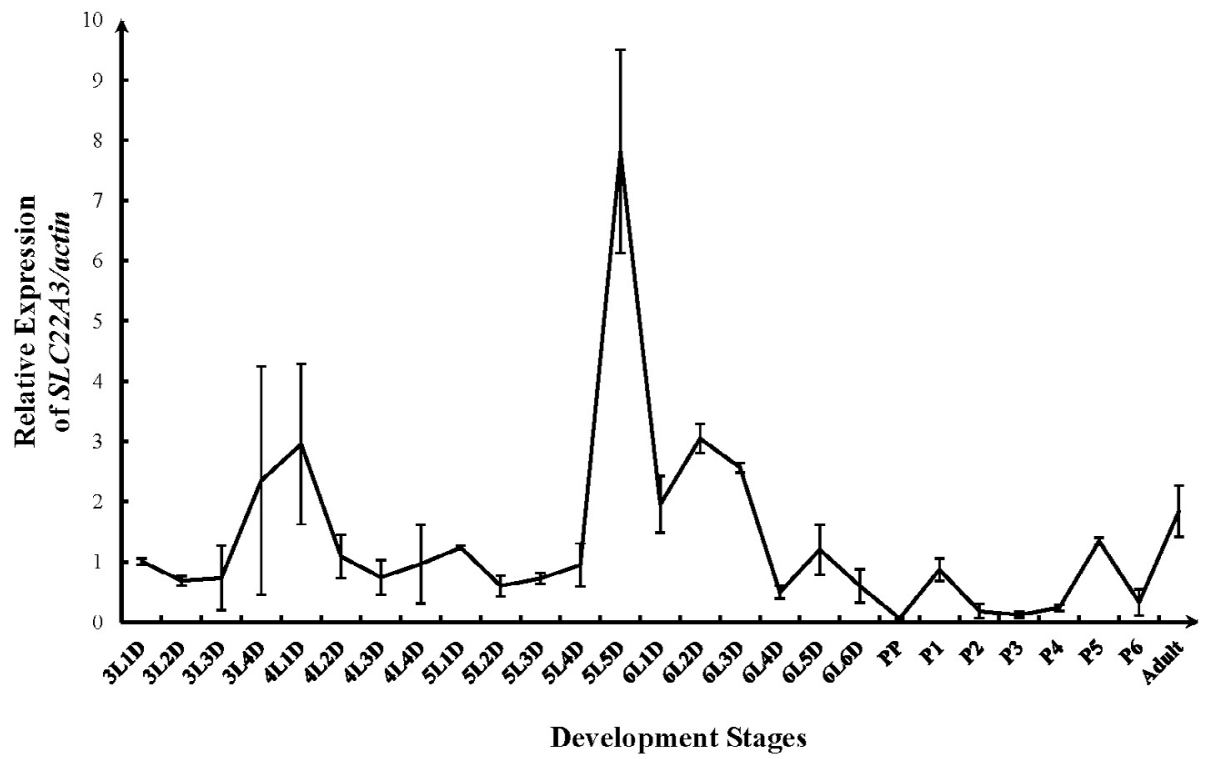

Fig. 4. The expression profile of CSuSLC22A3 gene during larva, pupa and adult development. The results indicated that this gene were highly expressed before molt. 
a

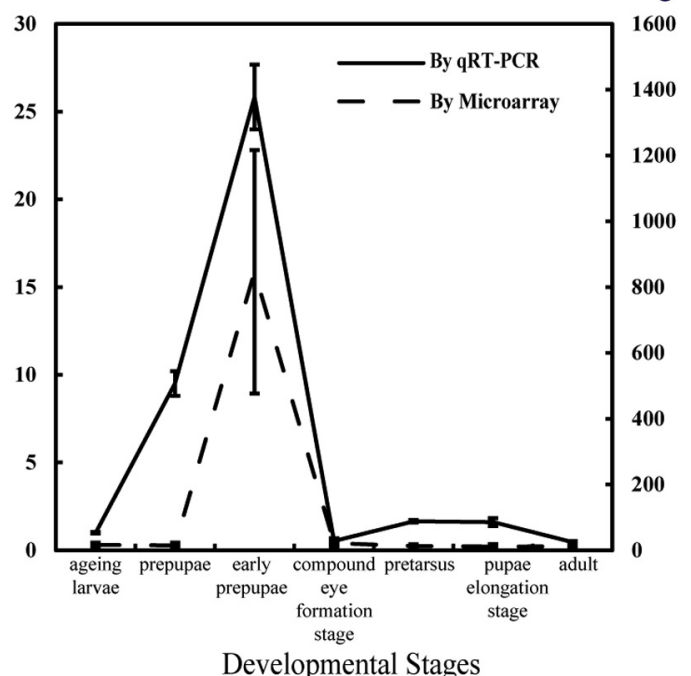

b

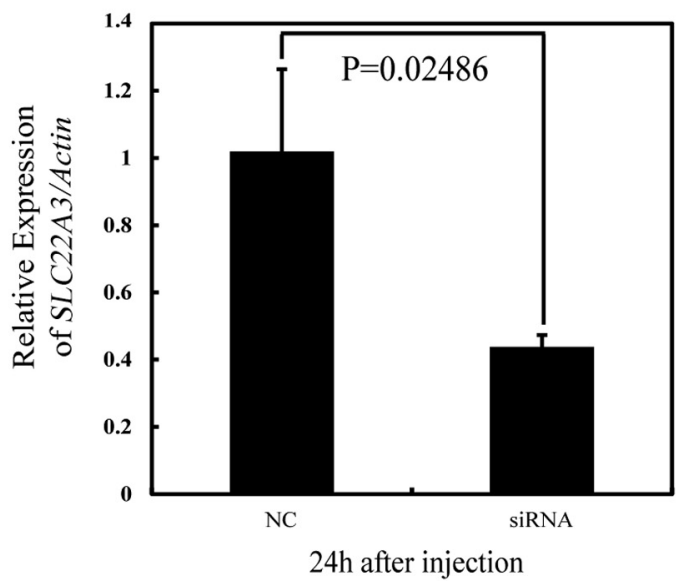

$\mathrm{C}$

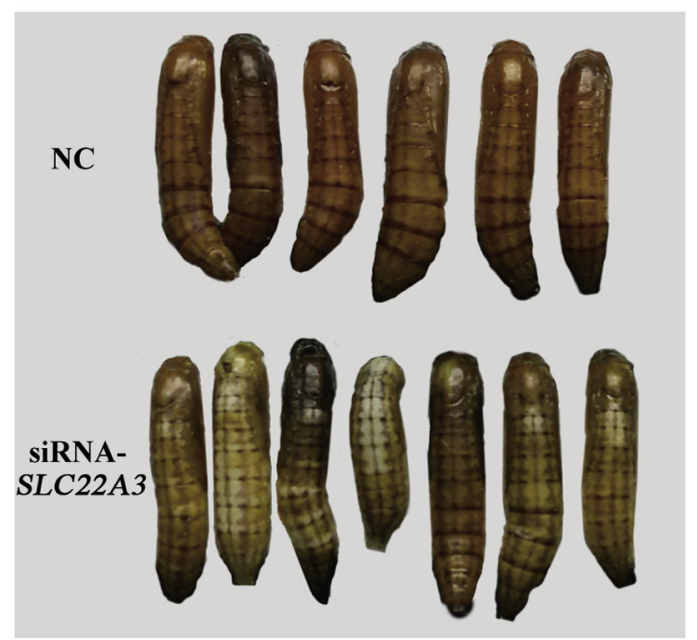

Fig. 5. RNA interference of CsuSLC22A3 induced a soft and milky pupa. (a) qPCR validation of SLC22A3 gene. (a) The microarray signal values of CsuSLC22A3 genes. (b) Relative expression after injection with siRNA (T-test, one-tailed, pvalue $=0.025$ ). (c) Abnormal phenotype of RNAi-treated individuals. Thirty insects were used for each treatment. All experiments were conducted in triplicate.
RNAi-treated individuals was only $40 \%$ of the control level (T-test, $\mathrm{p}<0.05$ ) (Fig. 5b). Knockdown of SLC22A3 gene led to deficiency in skeletonisation and pigmentation. A total of $90 \%$ of the RNAitreated individuals showed abnormal phenotypes of minor stiffening at 24-48 h after pupation, resulting in a white and soft epidermis on the abdomen, back and head. Then the pupa shrivelled and died one week after RNAi treatment (Fig. 5c). This result suggests that SLC22A3 has an essential role in pupal pigmentation and stiffness.

\section{Discussion}

Pupa is a unique stage in the life cycle of holometabolous insects, in which the larval tissues disintegrate and the adult organs appear (Buszczak and Segraves, 2000; Truman and Riddiford, 1999). Studying gene regulation in pupal development should be helpful for better understanding programmed cell death and adult organ formation. It is also useful to find environmental friendly targets for RNAi pest control since metamorphosis exists only in insects and other several animal groups (De Loof et al., 2014; Kaneko and Hiruma, 2014). Though the molecular mechanism of insect metamorphosis has been well studied in larvae, less studies have been carried out in C. suppressalis. Here, we identified 622 DEGs during pupation, pupa development and eclosion of SSB, which add the knowledge of genes associated with insect metamorphosis. The results indicated that the genes associated with substance metabolism, protein degradation, and cuticle digestion were highly expressed in the early pupal stages, whereas genes associated with signal transduction organisation were highly expressed in the late pupal stages, indicating that the active biological processes in the early pupal stages were different from those in the late pupal stages. Uncovering the roles of these genes may provide new insights into programmed cell death, cell proliferation, and tissue stem cells.

Among these DGEs, a high number of SLC genes were highly expressed during pupal development. The SLC gene family codes for membrane transport proteins that are responsible for transporting a variety of solutes, including charged and uncharged organic molecules, inorganic ions, and ammonia (Cheeseman, 2009). It has been reported that SLC6 acts as a nutrient-related transporter (Meleshkevitch et al., 2009; Miller et al., 2008; Rudnick et al., 2014). Mutations of SLC2A9 were responsible for sex-linked translucent larval skin of the silkworm (Cheeseman, 2009; Kiuchi et al., 2011). Thus, we silenced SLC22A3 in the early pupa of SSB, which led to an abnormal pupal epidermis. A similar phenotype appeared when $\mathrm{N}$ - $\beta$-alanyldopamine (NBAD) was decreased in the epidermis of the Mediterranean fruit fly (Wappner et al., 1995b). In B. mori, disrupting the biogenesis of NBAD resulted in white pupa (Kiuchi et al., 2011; Wappner et al., 1995a) and melanic pupa (Dai et al., 2015; Wappner et al., $1996 a, b)$. Since the SLC22A3 gene is an organic anion transporter (Koehler et al., 1997), we hypothesized that SLC22A3 might be involved in transporting NBAD or its precursor into the cuticle membrane, which requires further validation.

We identified a set of genes involved in larval-pupal and pupaladult transition. It has been reported that complete loss of $\mathrm{JH}$ in mod-mutated silkworms induced precocious metamorphosis with fewer larval-larval moults, yielding small pupa and small adults (Daimon et al., 2012). Since precocious metamorphosis significantly shortens the larval stage and reduces yield loss, it is possible to control insect pests by inducing pupation at the early larval 
stage. This method can control the pest without destroying the insect population and would be an ecologically friendly strategy. The identified 622 DGEs would be helpful in finding safe and efficient targets for RNAi pest control.

\section{Acknowledgements}

We thank professor Yoonseong Park for his critical comments on the manuscript. This work was supported by the National Key Research and Development Program [2016YFC1200600] and the National Science Foundation of China (31260431, 31301691).

\section{Appendix A. Supplementary data}

Supplementary data associated with this article can be found, in the online version, at http://dx.doi.org/10.1016/j.jinsphys.2016.12. 007.

\section{References}

Bolstad, B.M., Irizarry, R.A., Astrand, M., Speed, T.P., 2003. A comparison of normalization methods for high density oligonucleotide array data based on variance and bias. Bioinformatics 19, 185-193.

Buszczak, M., Segraves, W.A., 2000. Insect metamorphosis: out with the old, in with the new. Curr. Biol. 10, R830-R833.

Cheeseman, C., 2009. Solute carrier family 2, member 9 and uric acid homeostasis. Curr. Opin. Nephrol. Hypertens. 18, 428-432.

Cho, K.H., Daubnerova, I., Park, Y., Zitnan, D., Adams, M.E., 2014. Secretory competence in a gateway endocrine cell conferred by the nuclear receptor betaFTZ-F1 enables stage-specific ecdysone responses throughout development in Drosophila. Dev. Biol. 385, 253-262.

Colgan, T.J., Carolan, J.C., Bridgett, S.J., Sumner, S., Blaxter, M.L., Brown, M.J., 2011. Polyphenism in social insects: insights from a transcriptome-wide analysis of gene expression in the life stages of the key pollinator, Bombus terrestris. BMC Genomics 12, 623

Dai, F., Qiao, L., Cao, C., Liu, X., Tong, X., He, S., Hu, H., Zhang, L., Wu, S., Tan, D., Xiang, Z., Lu, C., 2015. Aspartate decarboxylase is required for a normal pupa pigmentation pattern in the silkworm, Bombyx mori. Sci. Rep. 5, 10885.

Daimon, T., Kozaki, T., Niwa, R., Kobayashi, I., Furuta, K., Namiki, T., Uchino, K., Banno, Y., Katsuma, S., Tamura, T., Mita, K., Sezutsu, H., Nakayama, M., Itoyama, K., Shimada, T., Shinoda, T., 2012. Precocious metamorphosis in the juvenile hormone-deficient mutant of the silkworm, Bombyx mori. PLoS Genet. 8, e1002486.

De Loof, A., De Haes, W., Janssen, T., Schoofs, L., 2014. The essence of insect metamorphosis and aging: electrical rewiring of cells driven by the principles of juvenile hormone-dependent $\mathrm{Ca}(2+)$-homeostasis. Gen. Comp. Endocrinol. 199, 70-85.

Deng, H., Zhang, J., Li, Y., Zheng, S., Liu, L., Huang, L., Xu, W.H., Palli, S.R., Feng, Q., 2012. Homeodomain POU and Abd-A proteins regulate the transcription of pupal genes during metamorphosis of the silkworm, Bombyx mori. Proc. Natl. Acad. Sci. USA 109, 12598-12603.

Eisen, M.B., Spellman, P.T., Brown, P.O., Botstein, D., 1998. Cluster analysis and display of genome-wide expression patterns. Proc. Natl. Acad. Sci. USA 95, 14863-14868.

Grabherr, M.G., Haas, B.J., Yassour, M., Levin, J.Z., Thompson, D.A., Amit, I., Adiconis, X., Fan, L., Raychowdhury, R., Zeng, Q., Chen, Z., Mauceli, E., Hacohen, N., Gnirke, A., Rhind, N., di Palma, F., Birren, B.W., Nusbaum, C., Lindblad-Toh, K., Friedman, N., Regev, A., 2011. Full-length transcriptome assembly from RNA-Seq data without a reference genome. Nat. Biotechnol. 29, 644-652.

Guo, Y., Guo, H., Zhang, L., Xie, H., Zhao, X., Wang, F. Li, Z., Wang, Y., Ma, S., Tao, J., Wang, W., Zhou, Y., Yang, W., Cheng, J., 2005. Genomic analysis of anti-hepatitis $\mathrm{B}$ virus (HBV) activity by small interfering RNA and lamivudine in stable HBVproducing cells. J. Virol. 79, 14392-14403.

He, Y., Zhang, J., Gao, C., Su, J., Chen, J., Shen, J., 2013. Regression analysis of dynamics of insecticide resistance in field populations of Chilo suppressalis (Lepidoptera: Crambidae) during 2002-2011 in China. J. Econ. Entomol. 106, 1832-1837.

Irizarry, R.A., Bolstad, B.M., Collin, F., Cope, L.M., Hobbs, B., Speed, T.P., 2003a. Summaries of Affymetrix GeneChip probe level data. Nucleic Acids Res. 31, e15.

Irizarry, R.A., Hobbs, B., Collin, F., Beazer-Barclay, Y.D., Antonellis, K.J., Scherf, U. Speed, T.P., 2003b. Exploration, normalization, and summaries of high density oligonucleotide array probe level data. Biostatistics 4, 249-264.

Jindra, M., Palli, S.R., Riddiford, L.M., 2013. The juvenile hormone signaling pathway in insect development. Annu. Rev. Entomol. 58, 181-204.

Jones, G., Jones, D., Li, X., Tang, L., Ye, L., Teal, P., Riddiford, L., Sandifer, C., Borovsky, D., Martin, J.R., 2010. Activities of natural methyl farnesoids on pupariation and metamorphosis of Drosophila melanogaster. J. Insect Physiol. 56, 1456-1464.

Kaneko, Y., Hiruma, K., 2014. Short neuropeptide F (sNPF) is a stage-specific suppressor for juvenile hormone biosynthesis by corpora allata, and a critical factor for the initiation of insect metamorphosis. Dev. Biol. 393, 312-319.
Kayukawa, T., Murata, M., Kobayashi, I., Muramatsu, D., Okada, C., Uchino, K. Sezutsu, H., Kiuchi, M., Tamura, T., Hiruma, K., Ishikawa, Y., Shinoda, T., 2014. Hormonal regulation and developmental role of Kruppel homolog 1, a repressor of metamorphosis, in the silkworm Bombyx mori. Dev. Biol. 388, 48-56.

King-Jones, K., Charles, J.P., Lam, G., Thummel, C.S., 2005. The ecdysone-induced DHR4 orphan nuclear receptor coordinates growth and maturation in Drosophila. Cell 121, 773-784.

Kiuchi, T., Banno, Y., Katsuma, S., Shimada, T., 2011. Mutations in an amino acid transporter gene are responsible for sex-linked translucent larval skin of the silkworm, Bombyx mori. Insect Biochem. Mol. Biol. 41, 680-687.

Koehler, M.R., Wissinger, B., Gorboulev, V., Koepsell, H., Schmid, M., 1997. The two human organic cation transporter genes SLC22A1 and SLC22A2 are located on chromosome 6q26. Cytogenet. Cell Genet. 79, 198-200.

Li, L.T., Zhu, Y.B., Ma, J.F., Li, Z.Y., Dong, Z.P., 2013. An analysis of the Athetis lepigone transcriptome from four developmental stages. PLoS One 8, e73911.

Meleshkevitch, E.A., Robinson, M., Popova, L.B., Miller, M.M., Harvey, W.R., Boudko, D.Y., 2009. Cloning and functional expression of the first eukaryotic Na+tryptophan symporter, AgNAT6. J. Exp. Biol. 212, 1559-1567.

Miller, M.M., Popova, L.B., Meleshkevitch, E.A., Tran, P.V., Boudko, D.Y., 2008. The invertebrate $\mathrm{B}(0)$ system transporter, D. melanogaster NAT1, has unique $\mathrm{d}$ amino acid affinity and mediates gut and brain functions. Insect Biochem. Mol Biol. 38, 923-931.

Ou, J., Deng, H.M., Zheng, S.C., Huang, L.H., Feng, Q.L., Liu, L., 2014. Transcriptomic analysis of developmental features of Bombyx mori wing disc during metamorphosis. BMC Genomics 15, 820.

Parthasarathy, R., Tan, A., Bai, H., Palli, S.R., 2008. Transcription factor broad suppresses precocious development of adult structures during larval-pupal metamorphosis in the red flour beetle, Tribolium castaneum. Mech. Dev. 125 299-313.

Pelis, R.M., Wright, S.H., 2014. SLC22, SLC44, and SLC47 transporters-organic anion and cation transporters: molecular and cellular properties. Curr. Top. Membr. 73, 233-261.

Pfaffl, M.W., 2001. A new mathematical model for relative quantification in realtime RT-PCR. Nucleic Acids Res. 29, e45.

Qi, L., Fang, Q., Zhao, L., Xia, H., Zhou, Y., Xiao, J., Li, K., Ye, G., 2016. De novo assembly and developmental transcriptome analysis of the small white butterfly Pieris rapae. PLoS One 11, e0159258.

Reza, A.M., Kanamori, Y., Shinoda, T., Shimura, S., Mita, K., Nakahara, Y., Kiuchi, M., Kamimura, M., 2004. Hormonal control of a metamorphosis-specific transcriptional factor Broad-Complex in silkworm. Comp. Biochem. Physiol. B: Biochem. Mol. Biol. 139, 753-761.

Riddiford, L.M., 1972. Juvenile hormone in relation to the larval-pupal transformation of the cecropia silkworm. Biol. Bull. 142, 310-325.

Riddiford, L.M., 2007. The control of metamorphosis in the kissing bug. J. Exp. Biol 210, 3133-3134.

Riddiford, L.M., 2008. Juvenile hormone action: a 2007 perspective. J. Insect Physiol. 54, 895-901.

Riddiford, L.M., 2012. How does juvenile hormone control insect metamorphosis and reproduction? Gen. Comp. Endocrinol. 179, 477-484.

Riddiford, L.M., Truman, J.W., Mirth, C.K., Shen, Y.C., 2010. A role for juvenile hormone in the prepupal development of Drosophila melanogaster. Development 137, 1117-1126.

Rudnick, G., Kramer, R., Blakely, R.D., Murphy, D.L., Verrey, F., 2014. The SLC6 transporters: perspectives on structure, functions, regulation, and models for transporter dysfunction. Pflugers Arch. 466, 25-42.

Santhanam, A., Liang, S.Y., Chen, D.Y., Chen, G.C., Meng, T.C., 2013. Midgut-enriched receptor protein tyrosine phosphatase PTP52F is required for Drosophila development during larva-pupa transition. FEBS J. 280, 476-488.

Schmitt-Engel, C., Schultheis, D., Schwirz, J., Strohlein, N., Troelenberg, N., Majumdar, U., Dao, V.A., Grossmann, D., Richter, T., Tech, M., Donitz, J. Gerischer, L., Theis, M., Schild, I., Trauner, J., Koniszewski, N.D., Kuster, E., Kittelmann, S., Hu, Y., Lehmann, S., Siemanowski, J., Ulrich, J., Panfilio, K.A. Schroder, R., Morgenstern, B., Stanke, M., Buchhholz, F., Frasch, M., Roth, S. Wimmer, E.A., Schoppmeier, M., Klingler, M., Bucher, G., 2015. The iBeetle largescale RNAi screen reveals gene functions for insect development and physiology. Nat. Commun. 6, 7822.

Stilwell, G.E., Nelson, C.A., Weller, J., Cui, H., Hiruma, K., Truman, J.W., Riddiford, L. M., 2003. E74 exhibits stage-specific hormonal regulation in the epidermis of the tobacco hornworm, manduca sexta. Dev. Biol. 258, 76-90.

Sultan, A.R., Oish, Y., Ueda, H., 2014. Function of the nuclear receptor FTZ-F1 during the pupal stage in Drosophila melanogaster. Dev. Growth Differ. 56, 245-253.

Truman, J.W., Riddiford, L.M., 1999. The origins of insect metamorphosis. Nature 401, 447-452.

Truman, J.W., Riddiford, L.M., 2007. The morphostatic actions of juvenile hormone Insect Biochem. Mol. Biol. 37, 761-770.

Tusher, V.G., Tibshirani, R., Chu, G., 2001. Significance analysis of microarrays applied to the ionizing radiation response. Proc. Natl. Acad. Sci. USA 98, 5116 5121.

Wang, X., Xiong, M., Lei, C., Zhu, F., 2015. The developmental transcriptome of the synanthropic fly Chrysomya megacephala and insights into olfactory proteins. BMC Genomics 16, 20.

Wappner, P., Hopkins, T.L., Kramer, K.J., Cladera, J.L., Manso, F., Allue, L.Q., 1996a Role of catecholamines and P-alanine in puparial color of wild-type and melanie mutants of the Mediterranean (Ceratitis capitata). J. Insect Physiol. 42, 455-461.

Wappner, P., Kramer, K.J., Hopkins, T.L., Allue, L.Q., 1996b. N-P-alanyldopamine metabolism for puparial tanning in wild-type and mutant niger strains of the 
Mediterranean Fruit Fly, Ceratitis capitata. Insect Biochem. Mol. Biol. 26, 585592.

Wappner, P., Kramer, K.J., Hopkins, T.L., Merritt, M., Schachtner, J., Allue, L.Q., 1995a White pupa: a Ceratitb3 capitata mutant lacking catecholamines for tanning the puparium. Insect Biochem. Mol. Biol. 25, 365-373.

Wappner, P., Kramer, K.J., Hopkins, T.L., Merritt, M., Schaefer, J., Quesada-AlluÉ, L.A., 1995b. White pupa a Ceratitis capitata mutant lacking catecholamines for tanning the puparium. Insect Biochem. Mol. Biol. 25.

Yamanaka, N., Rewitz, K.F., O'Connor, M.B., 2013. Ecdysone control of developmental transitions: lessons from Drosophila research. Annu. Rev. Entomol. 58, 497-516.

Yin, C., Liu, Y. Liu, J., Xiao, H., Huang S., Lin, Y, Han, Z, Li, F, 2014 ChiloDB: genomic and transcriptome database for an important rice insect pest Chilo suppressalis. Database (Oxford) 2014.
Yin, C., Shen, G., Guo, D., Wang, S., Ma, X., Xiao, H., Liu, J., Zhang, Z., Liu, Y., Zhang, Y., Yu, K., Huang, S., Li, F., 2016. InsectBase: a resource for insect genomes and transcriptomes. Nucleic Acids Res. 44, D801-D807.

Yin, V.P., Thummel, C.S., 2005. Mechanisms of steroid-triggered programmed cell death in Drosophila. Semin. Cell Dev. Biol. 16, 237-243.

Zhang, P., Aso, Y., Jikuya, H., Kusakabe, T., Lee, J.M., Kawaguchi, Y., Yamamoto, K., Banno, Y., Fujii, H., 2007a. Proteomic profiling of the silkworm skeletal muscle proteins during larval-pupal metamorphosis. J. Proteome Res. 6, 2295-2303.

Zhang, Y., Huang, J., Jia, S., Liu, W., Li, M., Wang, S., Miao, X., Xiao, H., Huang, Y., 2007b. SAGE tag based cDNA microarray analysis during larval to pupal development and isolation of novel cDNAs in Bombyx mori. Genomics 90, 372-379.

Zhang, Z., Yin, C., Liu, Y., Jie, W., Lei, W., Li, F., 2014. IPathCons and iPathDB: an improved insect pathway construction tool and the database. Database (Oxford) 2014 